\title{
Visualized analysis of the trend and hotspots in microbial spatial ecology: a bibliometric study from 2000 to 2020
}

\section{Sarfraz Hussain}

Nanjing Agricultural University

Hao Liu

Nanjing Agricultural University

Rabia Sabir

Nanjing Agricultural University

Muzammil Hassan Khan

Nanjing Agricultural University

Hui Cao ( $\nabla$ hcao@njau.edu.cn )

Nanjing Agricultural University https://orcid.org/0000-0003-3406-3803

\section{Research Article}

Keywords: Spatial Ecology, Biogeography, Diversity, Bibliometric, Citespace

Posted Date: February 17th, 2022

DOI: https://doi.org/10.21203/rs.3.rs-1331467/v1

License: (a) (i) This work is licensed under a Creative Commons Attribution 4.0 International License. Read Full License 


\section{Abstract}

Spatial ecology is a broad-spectrum term used in a variety of studies depending on the subdiscipline of life. Spatial ecological studies focus on patterns and interactions within and between species at the local, regional and global scales. The scientific literature published on spatial microbial ecology is ample and covers a wide array of objectives, including biogeographic patterns of microorganisms, international collaboration, hot topics, keywords and productive journals. We used a science mapping approach to provide a global and systematic bibliometric synthesis in this area. A total of 7022 publications on spatial microbial ecology were retrieved from the Web of Science database in 2000-2020. Temporal analyses showed a rapid growth in publications and citations noted after 2010. Results based on cluster analysis revealed that the top productive countries in this area belong to developed countries other than China. Keyword analysis showed that diversity, bacteria and dynamics are research hotspots in this field. The citation burst indicated that researchers focused on traditional techniques in the early stage, while new techniques and models were developed in the later stage. These findings suggest that spatial microbial ecology is still in a developmental stage. This study provides a systematic understanding of the development and panoramic road map for conducting future research in spatial microbial ecology.

\section{Introduction}

The main purpose of ecology is to understand how biodiversity is established and maintained. Spatial ecology is a fundamental ecological field for investigating the mechanisms that give rise to spatial patterning in the configuration of the population. This field is usually referred to as landscape ecology for macro-organisms, for which a wealth of conceptual structures, analytical processes and models have been developed [1]. In a microbial community, spatial patterning is possibly a consequence of several complex processes. Spatial dynamics of species diversity provide information on biodiversity-regulating processes and are essential in the development of conservation priorities. Ecosystem spatial complexity enables the coexistence of organisms and the processes they conduct and occurs at diverse scales, varying from landscapes to soil pores micrometres in size [2-4]. Microbial populations' spatial configuration has significant implications for their evolution. Microbes living in subdivided populations are vulnerable to a greater risk of extinction and diversity-purging community bottlenecks. They are capable of adapting to local environmental conditions, and these local interactions between intra- and inter-species create a mosaic ecosystem that can promote coevolution and induce diversification of microbes $[5,6]$. These interactions play an important role within and among local communities in the evolution of microbial diversity $[7,8]$.

Brock (1987) proposed that spatial research would rely on scales relevant to individuals, for example bacterial cells, in order to research the ecology of microorganisms adequately. He mentioned that this is the 'only way we can really see organisms in their actual environments' and through evolutionary studies, 'without knowing where these organisms lived, (how) we can make any sense'. Since there are usually about $10^{6}$ bacteria in a single millimetre of unpolluted surface water, and soils can contain up to $10^{10}$ microorganisms in a single gram, it is a challenge to research microbial ecology at the level of an 
individual [9]. Most environmental scientists are mainly interested in how microbial interactions are manifested on larger spatial scales and how nutrient cycling, decomposition, primary productivity, and other microbially mediated ecosystem functions are regulated on human-relevant scales by this activity [10]. Medical microbiology emphasizes the investigation of the spatial distribution of microbial communities in different organs, including the tongue dorsum [11], gastrointestinal tract [12], skin [13] and other organs [14-17].

An advance and important tool used to evaluate research activity on a certain topic is bibliometrics evaluation, which is described as the use of mathematical methods for analysing articles published in terms of quality and quantity $[18,19]$. Information about publication progress, global collaboration, top active countries, organisations and authors are described in bibliometric review. Furthermore, articles that publish on the subject of interest are also presented. Maps are used as a tool of visualization in bibliometric studies. Through the use of databases such as PubMed, Google Scholar, Scopus or the Web of Science, it is possible to download published papers on a certain topic [20,21]. Over the past years, bibliometrics has been widely used to analyse scientific research in host-microbe interactions, functional-gene-containing microbial communities, pathogenic microbes and microbial biodiversity [2227]. However, to the best of our knowledge so far, no bibliometric studies have assessed the spatial heterogeneity of microbial communities at the global level. In this review, we aimed to comprehensively analyse the status of spatial microbial ecology research as emerging categories and their research trends with a bibliometric and visual approach. Such an approach serves to provide a systematic, comprehensive bibliometric overview of the literature on spatial microbial ecology and trends in microbial ecology.

\section{Materials And Methods}

\subsection{The data acquisition and query criteria}

A bibliometric study depends upon databases in the literature. The Web of Science Core Collection (WoSCC) database is among the best-known datasets, a curated selection of high-quality academic material on the Web of Science ${ }^{\mathrm{TM}}$ platform (WoS; previously known as Web of Knowledge), an online, subscription-based scientific citation indexing service maintained by Thomson Reuters [28]. The WoSCC database remains open at all times, so to prevent bias related to regular database changes, we conducted the WoSCC literature retrieval on a single day, February 11, 2021.The synonyms for spatial ecology and microbial community were included in the search strategy as follows: TS $=\left(\left(\left(()\left(\left(\left(\right.\right.\right.\right.\right.\right.$ Spatial ecology $\left.{ }^{\star}\right)$ OR (Spatial heterogeneity*) OR (Spatial diversity*)))) AND (((((Microbial*) OR (Prokaryotic*) OR (bacteria*) OR (fungi*) OR (Microbiota*) OR (Archaea*)))))))))). In the research query, the asterisk marks were used to increase comprehensiveness.

\subsection{Refining the retrieved documents}

The data retrieval scheme and process of the current study are shown in (Fig. 1). Briefly, the documents retrieved were restricted to journal research articles and review documents. Proceeding papers, book 
chapters, early access articles, editorial materials, data papers and retracted papers were excluded. The main objective of this step was to emphasize original research data as compared with scientific publications in other formats. The study period selection was based on two main criteria: first, fewer than 100 documents per year were published between 1985 and 2000, and second, global concern regarding spatial microbial ecology has developed in the last two decades. We restricted our search to the English language because English is an international language, and research material presented in English is easily understood by the entire research community.

\subsection{Analysis of exported data}

The data from all 7022 selected documents were exported in html format for further analysis. The basic bibliometric analysis, including top journals, highly cited top authors, top cited and publishing countries and publication record per year, was conducted in IMB SPSS Statistics 25.0 and Microsoft excel 2019. The citation report for evaluation of publication quality, including the total number of times cited, average citations per item and h-index, was created on WOSCC Thomson Reuters $[29,30]$. To create networks and visualize links, we used the VosViewer software developed by the Centre for Science and Technology Studies, Leiden University (http://www.vosviewer.com/), and Citespace [31]. In the visualisation of trends and patterns, CiteSpace is commonly used in the scientific literature. It incorporates functions such as the drawing of visual co-citation maps, the separation of co-citation networks, the identification of turning points, search of key nodes and the analysis of area evolution [32].

The document co-citation network, author co-citation network, citation and author bursts were performed with CiteSpace 5.7.4R in this analysis. The specific analytical parameters on CiteSpace were selected as follows: time slicing (from 2000 to 2020, years per slice = 1), term source (title, abstract, author, keyword and keywords plus), node type (cited reference and cited author were selected one by one). The link strength (cosine), the scope of the link (within slices), the criteria for selection (top 50 per slice) and pruning (none). The essential features of a citation burst were the intensity and duration of the burst. The burst detection over a specified period revealed abrupt changes in terms or citations, thereby identifying emerging trends in research [33].

Visualization of similarities (VOS) mapping was used to measure and locate each topic in a twodimensional network in such a way that the difference among two items showed the relatedness or similarity as precisely as possible. The VOS clustering approach was implemented in different clusters, which were labelled with different colours $[34,35]$. In the results portion, the interpretation of the data visualization is explained in detail. The general interpretation is as follows: the size of the circles and the label font represent the number of occurrences, the colours of the circles represent the type of clusters, and the distance between the two circles shows the relatedness and similarity between the circles [36, 37]. The normalization association method was selected in the layout. All visualization images contain the top 500 items in order to attain appropriate visualization [37]. All keywords were selected in cooccurrence analysis, and a total of 808 keywords were retrieved. The minimum number of keyword occurrences was set to 20 , and the top 500 keywords were included from the threshold for visualization. 


\section{Results}

\section{Spatiotemporal trends in spatial microbial ecology research publication and citation}

Our search based on 'topic' showed a total 7627 publications between 2000 and 2020, and we retrieved 7022 documents after applying the exclusion and inclusion criteria, including 6450 (91.85\%) articles and $572(8.14 \%)$ reviews (Fig. 1). The temporal trend in the number of publications and the citation rate in the field of spatial microbial ecology from 2000 to 2020 (Fig. 2A) revealed a steady increase, with an accelerated rate after 2005 and a total of approximately 334.4 publications per year. We further divided the publication of documents into two temporal stages, from 2000 to 2010 and 2011 to 2020 . The initial period showed low growth with an average 165 publications per year, while the later period showed an impressive trend of 520.8 publications per year. The 7022 retrieved documents received 252,980 citations, an average of 36.026 citations per item, with an h-index of 192. The temporal distribution of citations (Fig. 2B) indicated an average of 12,046.66667 citations per year. The spatial contribution of research in spatial ecology revealed that United States of America (USA) has dominated the contribution to this field with 2508 publications and 126,127 citations. Peoples Republic of China (PRC) ranked second with respect to publications, while Germany ranked second in term of citations. The worldwide publication and citation trends are shown in (Fig. S1).

Based on the authors' affiliations in the same publication as evidence of production and cooperation, an assessment of the contribution of various countries to the development of spatial microbial ecology studies was performed (Fig. S2). The nodes are the number of authors from the same region, and the interest of the country is the diameter of the node. The presence of an arc between the nodes indicates collaboration among researchers from different countries. The cluster reveals that scientific work occurred through established collaborations. In international cooperative partnerships in spatial microbial ecology research, the USA is perhaps the dominant country and holds a key role in the figure. The USA, China and Germany have played an important role in supportive collaboration among nations, with the majority of publications. The USA cooperated frequently with China, Germany, England, Japan and Canada. Among them, the US-China collaborative partnership was predominant and ranked first with 238 collaborative publications. China also cooperated effectively with other nations, such as Australia, England and Germany with 58, 52 and 44 publications, respectively.

\section{Author productivity and collaboration in research}

Author productivity is a valuable indicator of the most active researchers in a given field. A collaboration map illustrating the co-author network of the top 100 researchers is shown in (Fig. 3A). Each node represents an author, where the size of the node represents the number of documents authored by that author, the colour of the node represents the cluster to which the author belongs and the author-node relationship represents the author's co-authorship or research partnership. The cluster is a set of authornodes that are closely connected. The wider the author-node, the greater the number of documents the author has written. The number of co-authored documents by the author-nodes on both sides of the link, 
as well as the research cooperation between those authors, rises as the link grows thicker. The fact that the author-nodes are the same colour suggests that they are closely related.

After excluding authors with fewer than five publications, there were 503 authors contributing 3657 publications on spatial microbial ecology. There are 80 authors in this field who have published more than ten articles. The top three most prolific authors in this field were Zhou, followed by Xie, Tedersoo, and Soininen, with 42, 27, 26 and 26 publications, respectively (Table S1). The co-citation analysis reveals that the work of Knight secured first position with 10,289 citations, followed by Fierer with 9382 total citations and Lauber with 7101 total citations in the second and third positions, respectively. Details of the top cited works are shown in (Fig. 3B). In addition, as shown in (Tab. S1), the average citation frequencies of these authors per publication were also in the top three, but in descending order: Lauber. (1420), Knight (489) and Fierer (426).

\section{Journal distribution}

From 2000 to 2020, a total of 1178 journals published articles on spatial ecology, with 5599 articles published in a diverse range of 270 journals after excluding journals with fewer than five publications (Fig. S3a). The top ten most productive journals (TP $>100$ ) published a total of 1867 articles $(33.34 \%)$. Frontiers in Microbiology, FEMS Microbiology Ecology and Plos One were the top three journals with the most publications (Table S2). In addition, we evaluated the citations received by these journals. (Fig. S3b). A list of the top ten journals with the highest numbers of citations is shown in (Table 1). Applied and Environmental Microbiology ranked first, indicating that it has a significant impact in this field. Similarly, Proceedings of the National Academy of Sciences of the United States of America and International Society of Microbial Ecology (ISME) ranked second and third, respectively, with over 9500 citations and high citation frequencies (Table 1).

\section{Contributions of institutes}

By analysing the distribution of the research institution where the author works, we can understand the scientific research capabilities and the research atmosphere of the institution. There were 397 different institutions worldwide showing research interest in spatial microbial ecology, so we set the threshold value for the total number of research institutions' publications at 10. Only five of these institutions ( $0.89 \%$ of the total) published more than 100 papers. We selected the top 100 institutes for network analysis as shown in (Fig. S4a). From these 100 selected institutes, the ten most highly productive institutes are listed in (Table S3). Among these institutes, four of them were from the USA, two were from China and one was from each of Spain, France, Finland and Denmark. The Chinese Academy of Sciences had the most publications (498), greatly outnumbering any other institution. The second research institute was University of Chinese Academy of Sciences, which had 166 publications. The third most productive research institute was the National Institute for Agricultural Research, which had 125 published articles. 
The US institutions continued to be the leaders in this field (Table S3). Furthermore, research universities accounted for six of the top ten most productive institutes, as well as seven of the top ten most citated institutes. This reflects the fact that research universities are at the forefront of this field. According to citation analysis, there were at least 2000 citations for 58 organizations, as shown in (Fig. S4b). The University of Colorado had the most citations $(14,845)$, followed by the Chinese Academy of Science and Stanford University, each of which had 8958 and 8791 citations.

\section{Co-citation analysis on cited authors}

The most often cited authors and relevant information may be retrieved from the author co-citation network based on the number of co-citation occurrences. The weight of the author's citations is reflected by the size of each node. The links reflect a cooperative relationship between two authors. This study used co-citation analysis to create a map of 1000 authors that consisted of five clusters, as shown in (Fig 4A). The top three positions in terms of total co-citation frequencies were secured by Fierer (1176), Edgar (1161) and Caporaso (1140 citations). Furthermore, author burst detection can determine which author has had the greatest influence over a given period. The top 24 authors' bursts are shown in (Fig. 4B). In the authors' citation burst detection analysis, the strongest burst belonged to author Xie with a strength of 6.91, followed by Deng and Noah. There was a burst of nine authors that lasted until 2020.

\section{Co-citation analysis of cited references}

The analysis of references is an important aspect of bibliometrics-based visualization, as it reflects the field's research database. In addition, it is also an important source for exploring the research context and direction of development [38]. We used CiteSpace to draw the cited reference map based on the cocitation network and to analyse the cited references in the field of spatial microbial ecology. The pruning algorithm consisted of the Pathfinder, pruning sliced networks and pruning the merged network. We were able to obtain the visual network after running Citespace. The key co-citation clusters on the network were labelled by titles, as shown in (Fig. 5). In this network, there were 1614 nodes and 3146 edges. The network density was 0.0059 . The Modularity $Q$ was $0.8017(Q>0.3)$, which generally indicates a significant clustering structure, and the Mean Silhouette was 0.898 , which were important criteria for evaluating the validity of the mapping. The $Q$ and $S$ values (Fig. 5) indicate that the clustering structure was significant and that the clustering result was reasonable. The network was divided into 17 clusters of co-citation networks, which were then labelled on their own citers by index terms. The top six clusters are summarized (Table 2). Briefly, the largest cluster (\#0) is a biogeographic pattern with 227 members and a silhouette value of 0.876 . The most active citer in this cluster is Jiao (2020). The second largest cluster (\#1), categorized as fungal communities, has 201 members and a silhouette value of 0.869 , with the majority of the references published in 2012. Griffiths (2011) is the most active citer in this cluster. The third largest cluster, named ectomycorrhizal fungi, has 194 members, and the active citer is Hang-wei (2013). It is worth noting that the highest ranked item by citation, burst and centrality belongs to cluster \#3. 
The timeline visualization of the references co-citation analysis network is shown in (Fig. S5). Timeline visualization analysis showed that clusters \#3, \#8 and \#13 are labelled as bacterial 16s, Picea abies and anaerobic process evaluation, respectively, indicating the beginning of work in this era. However, the longest clusters depicted in the timeline visualization is cluster \#8, labelled as Picea abies. Besides this, clusters \#0, cluster \#1 and cluster \#7, labelled as biogeographic pattern, fungal communities and spatial organization, respectively, are the most popular now, and they have been going on for many years.

In addition, citation bursts have been successfully applied to capture the sharp increase in a relevant research interest. The top 30 burst references generated by CiteSpace are shown in (Fig. S6). In the figure the dark blue line represents the time after publication, while the red line represents the beginning and ending time of the literature as a research hotspot. It also demonstrates that only five references had the strongest citation bursts before 2010, with Microbial biogeography: putting microorganisms on the map, published by Martiny coming first with a strength of 41.3 [39]. During this time, they were primarily concerned with microbial distribution. Following 2010, many of the references had the strongest citation bursts, in which QIIME, published by Caporaso with a strength of 53.12, allowed analysis of highperformance community sequence data [40]. The most recent reference with citation bursts appeared in 2018, and six references had a burst that lasted until 2020.

\section{Co-citation analysis of keywords}

The keywords are generalizations of the topics in the literature [41]. We performed an analysis of keywords that can be used to determine the hotspots in the field of spatial microbial ecology. It is also used to track hot topics and research trends [42]. A total of 808 keywords were found, eight of which have a frequency greater than 500. It shows the network of co-occurring terms, which includes 808 nodes and 4698 links. The node in the network represented a keyword, and its size depended on the frequency of cooccurrence. The keywords with high frequencies included diversity (2146), bacteria (747) and microbial community (662) (Fig. 6A).

Keyword burst detection also showed that many burst keywords emerged in 2000-2010 (Fig. 6B). The burst strength of $16 \mathrm{~s}$ ribosomal RNA was 29.45, and the burst in 16s ribosomal RNA research in 2000 lasted until 2011, suggesting that 16s ribosomal RNA is a hotspot in spatial microbial studies. This also corresponds to the keyword diversity, because studies on diversity rely on 16 s ribosomal RNA data. Prior to 2010 , researchers used basic biological techniques to study microbial ecology, including keywords like 'in situ hybridization', 'PCR', 'DGGE', 'T-RFLP', 'gradient gel electrophoresis', 'biome', 'spatial heterogeneity', 'community structure', 'geostatistics', 'gene' and 'assemblage'. After 2010, the application of some advanced methods and technologies, such as the keywords 'pyrosequencing', 'high-throughput sequencing' and 'database', has further advanced the research in this field.

\section{Discussion}

Spatial microbial ecology is carried out on a variety of scales, from the millimetre and particle-size spectrum to the centimetre and meter scale of plot-size fields, as well as landscape and geographic 
scales [43]. This study used two types of bibliometric visualization tools, such as Citespace and VOS viewer, to conduct the analyses in the field of spatial microbial ecology during the period 2000-2020 based on 7022 records retrieved from the WOS database. The main purpose of the current study was to create understanding about spatial microbial ecology. Different perspectives were examined, such as the contributions of different countries, productive journals in terms of publication and citation, active institutions, prolific authors, highly cited references and keyword distribution analysis to represent intellectual structures like influential studies and hot topics. The numbers of publications on selected topics increased substantially after 2005 , and most of the studies were published in a later stage, 20152019 , while a weaker publication record was found in 2020. We found that there was a gradual increase in the number of publications from 2000 to 2020, while there was rapid growth in this area after 2010, but we found a decrease in publications in 2020. Research in this field requires scientists to take samples from different regions, which requires travel around the world. This decline could be due to the COVID-19 outbreak, as several countries around the world have also taken corresponding measures, such as exit and entry restrictions [44].

The most productive author in this field was Zhou (China), with 38 publications, while the most cited author was Knight (USA). The most productive research institution was Chinese Academy of Sciences, but the most cited institution was Colorado University. The U.S. and China made important contributions in this research area, the former with productive publications in relevant journals, the latter with productive institutions and authors. Although China's institutions have published many documents, their citation rate is lower, which may be due to limitations in the development and application of basic bioinformatics software, while the U.S., on the other hand, still dominates in this field. However, about 238 articles on spatial microbial ecology have been published in China, with only 12 publications from 2000 to 2010 , while the remaining $95 \%$ were published after 2010 . These findings suggest recent development and scientist interest in this area. From a geographical point of view, most of the countries with more research in this area are in Europe, such as Germany, France and England, from the European region six countries are in the top ten productive countries. The third largest organization (INRA) has also participated in this area from Europe. Conducting research in this area requires a lot of data analysis, which inevitably requires the use of some advanced software and analytical tools, such as the use of Illumina HiSeq and MiSeq platforms for high-throughput sequencing [45, 46], the use of some R packages [47], mothur [48], QIIME [49] and FUNGuild [50] to analyse raw data. At the same time, these tools must be professionally trained and require a high investment of time and money. According to our findings, the ten countries with the largest number of documents, with the exception of China, are all developed countries, and most of them are in Europe, which shows that developed countries have a major role in this area. Economic and technical issues may be the key limitations affecting the rapid development of this field in developing countries [51].

It is possible to identify the most productive journals in a certain subject in order to assist researchers in selecting the most relevant journal. This can improve their ability to evaluate the literature in the field, broaden their academic horizons and help them achieve technological innovation [52]. In addition, identifying key journals can help scholars to submit and publish articles, illustrating their academic 
achievements. On the other hand, scientists may have many journal choices that make it difficult to select the most appropriate journal [53]. Our analysis indicated that seven of the most cited journals were also among the top ten most productive, with Applied and Environmental Microbiology ranking first, showing that it has a significant effect on the field. These findings indicated that research quality in this field still needs improvement, and international collaboration between researchers should be strengthened to produce high-quality work.

An upsurge in the frequency of a study's citation over a period of time is seen as a mark of academic focus, which implies an underlying research trend [54]. By tracking the temporal trends of burst references, we can easily understand the major research areas of the scientific community at some point in time [41]. The development of research hotspots in spatial microbial ecology was illustrated using burst detection analysis. Early studies focused on microbial distribution, such as the structure of bacterial communities at the continental scale [55-57], spatial heterogeneity $[58,59]$ and fundamental microbial processes like the soil microbial nitrogen cycle [60], nitrogen fixation [61], nitrification [62] and the carbon cycle [63]. In recent years, with the continuous advancement in technology, researchers are also constantly developing new research models and new analysis methods in this field [64], which are enabling the further development of this field. They began to focus on their interaction with their habitat, such as biotic and abiotic interactions [65], how these organisms are harnessed in human endeavours and their impact on society. Moreover, their effect on agricultural production, health and industry were studied $[66,67]$. Some studies focused on the effect of treatments on microbial community structure and the observed biodiversity patterns of microorganisms such as the effect of biochar on communities [68]. In the last decade, microbial ecologists have emphasized the development of technical and bioinformatics methods for studying the structure and assembly process of microbial communities, such as the use of quantitative PCR to determine the number of genomes of a specific microbe per unit volume of the sample [69], inferential ecological studies with multivariant analysis [70], Neutral-Theory-based process models, inferential ecological stochastic and/or deterministic processes by Null Model Analysis, which explain more detailed ecological processes compared with previous years.

\section{Conclusion}

To the best of our knowledge, this is first comprehensive bibliometric analysis to reveal extensive information about development and hot topics in the field of spatial microbial ecology. Steady growth was observed from 2000 to 2020 . The annual publication output had increased by about 7.5 times, and annual citation increased by about 696.5 times over 20 years. At the same time, in recent years, researchers have also published articles in journals with high IF, such as PNAS, ISME, NAT METHODS and NAT REV MICROBIOL. In addition, through the co-occurrence analysis of research countries and institutions, we found that most research is performed in developed countries and that these countries have cooperated closely with each other, while China, with great influence in recent years, is the only developing country. Furthermore, based on a co-occurrence analysis, we discovered that the majority of research is conducted in developed countries and that these countries collaborate closely with each other, while China is the only developing country with significant influence in recent years. Due to the lack of 
funds and technology, there is little research in developing countries. At present, most of the research focuses on methods and technology development. There are few studies on the spatial patterns of microorganisms and the process of community assembly. At the same time, there are few studies that report a wide range of microbial ecology. In addition, many studies are on surface microbial ecology, and there are few studies on deep microbial ecology. The purpose of technological development is its application. In the future, research will focus on the microbial assembly process and development of comprehensive models. This study will assist researchers who are interested in spatial microbial ecology to better understanding the field's development patterns. This analysis can also assist scientists to efficiently accessing the journals, authors, or publications to which they want to refer. In summary, the present study provides a comprehensive review of the trends and gaps in the field of spatial microbial ecology and thus lays the groundwork for future research.

\section{Study Limitations}

Although this is the first bibliometric study of spatial microbial ecology, there are some limitations, as follows: First, only English-language articles were selected for this study, thus excluding certain nonEnglish papers that could provide further information and more recent developments in spatial microbial ecology. Second, in this study, two types of publications (articles and reviews) were selected, and future studies might expand the scope of the data collection to include additional types of publications (e.g., proceedings papers, book chapters, early access). Furthermore, another limitation is related to new papers and works that are rarely cited. An additional limitation is that new articles and papers that are rarely cited are not included in the database.

\section{Declarations}

Author Contributions: Author Contributions: Writing-original draft preparation, S.H.; software, L.H.; Writing-review and editing, R.S., and M.H.K.; Supervision, H.C. All authors have read and agreed to the published version of the manuscript.

Funding: This work was financially supported by the National Natural Science Foundation of China $(41501279,42077026)$.

Data Availability Statement: All data supporting the findings of this study are available on request from the corresponding author (Hui Cao).

Acknowledgments: We thank Dr. Senlin Liu for their valuable advice on data analysis.

Conflicts of Interest: The authors declare that they have no competing interests.

\section{References}


1. Proctor, D. M., \& Relman, D. A. (2017). The Landscape Ecology and Microbiota of the Human Nose, Mouth, and Throat. Cell Host \& Microbe, 21(4), 421-432

2. a, C. H. E. (2002). and D.A.W. b, Spatial soil ecology.

3. Baldrian, P., \& Větrovský, T. (2012). Scaling Down the Analysis of Environmental Processes: Monitoring Enzyme Activity in Natural Substrates on a Millimeter Resolution Scale. Applied and Environmental Microbiology, 78(9), 3473-3475

4. Baldrian, P. (2014). Distribution of Extracellular Enzymes in Soils: Spatial Heterogeneity and Determining Factors at Various Scales. Soil Science Society of America Journal, 78(1), 11-18

5. Forde, S., Thompson, J., \& Bohannan, B. (2004). Adaptation varies through space and time in a coevolving host-parasitoid interaction. Nature, 431(7010), 841-844

6. KRIST. (2000). Spatial variation in susceptibility to infection in a snaill $\backslash u 2013$ trematode interaction. Parasitology, 121(4), 395-401

7. Vos, M., et al. (2009). Local Adaptation of Bacteriophages to Their Bacterial Hosts in Soil. Science, 325(5942), 833-833

8. Little, A. E. F., et al. (2008). Rules of Engagement: Interspecies Interactions that Regulate Microbial Communities. Annual Review of Microbiology, 62(1), 375-401

9. Brock, T. (1987). The study of microorganisms in situ: progress and problems. in Symp. Soc. Gen. Microbiol.

10. Franklin, R. B., \& Mills, A. L. (2007). The spatial distribution of microbes in the environment. Springer

11. Wilbert, S. A., Mark, J. L., Welch, \& Borisy, G. G. (2020).Spatial Ecology of the Human Tongue Dorsum Microbiome. Cell Reports, 30(12):4003-4015.e3

12. Li, N., et al. (2020). Spatial heterogeneity of bacterial colonization across different gut segments following inter-species microbiota transplantation. Microbiome, 8(1), 161

13. van Veelen, H. P. J., Salles, J. F., \& Tieleman, B. I. (2017). Multi-level comparisons of cloacal, skin, feather and nest-associated microbiota suggest considerable influence of horizontal acquisition on the microbiota assembly of sympatric woodlarks and skylarks. Microbiome, 5(1), 156

14. McMullen, C., et al. (2020). Topography of the respiratory tract bacterial microbiota in cattle. Microbiome, 8(1), 91

15. D'Amato, A., et al. (2020). Faecal microbiota transplant from aged donor mice affects spatial learning and memory via modulating hippocampal synaptic plasticity- and neurotransmissionrelated proteins in young recipients. Microbiome, 8(1), 140

16. Molinero, N., et al. (2019). The human gallbladder microbiome is related to the physiological state and the biliary metabolic profile. Microbiome, 7(1), 100

17. Glendinning, L., et al. (2017). Comparing microbiotas in the upper aerodigestive and lower respiratory tracts of lambs. Microbiome, 5(1), 145

18. Broadus, R. N. (1987). Toward a definition of "bibliometrics". Scientometrics, 12(5), 373-379

19. Okubo, Y. (1997). Bibliometric indicators and analysis of research systems: methods and examples. 
20. Macías-Chapula, C. A., \& Mijangos-Nolasco, A. (2002). Bibliometric analysis of AIDS literature in Central Africa. Scientometrics, 54(2), 309-317

21. Ramos, J. M., et al. (2008). A bibliometric analysis of tuberculosis research indexed in PubMed, 1997-2006. Int J Tuberc Lung Dis, 12(12), 1461-1468

22. Costa, C., et al. (2020). Global Deep-Sea Biodiversity Research Trends Highlighted by Science Mapping Approach.Frontiers in Marine Science, 7(384)

23. Sweileh, W. M., Moh'd, A., \& Mansour (2020). Bibliometric analysis of global research output on antimicrobial resistance in the environment (2000-2019). Global Health Research and Policy, 5(1), 37

24. Liu, Y., Wu, K., \& Zhao, R. (2020). Bibliometric analysis of research on soil health from 1999 to 2018. Journal of Soils and Sediments, 20(3), 1513-1525

25. Wang, Z., Huang, C., \& Li, X. (2020). Research Trends and Hotspot Analysis of Conjunctival Bacteria Based on CiteSpace Software. BioMed Research International, 2020, 2580795

26. Nsenga Kumwimba, M., et al. (2020). Anammox-based processes: How far have we come and what work remains? A review by bibliometric analysis. Chemosphere, 238, 124627

27. Zheng, M., Fu, H. Z., \& Ho, Y. S. (2017). Research trends and hotspots related to ammonia oxidation based on bibliometric analysis. Environmental Science and Pollution Research, 24(25), 2040920421

28. Wáng, Y. X. J., et al. (2014). Implications of Web of Science journal impact factor for scientific output evaluation in 16 institutions and investigators' opinion. Quantitative Imaging in Medicine and Surgery, 4(6), 453-461

29. Sweileh, W. M., et al. (2015). Public, environmental, and occupational health research activity in Arab countries: bibliometric, citation, and collaboration analysis. Arch Public Health, 73(1), 1

30. Sweileh, W. M., et al. (2014). Bibliometric analysis of nutrition and dietetics research activity in Arab countries using ISI Web of Science database. Springerp/us, 3, 718

31. Chen, C. (2004). Searching for intellectual turning points: Progressive knowledge domain visualization. Proceedings of the National Academy of Sciences, 101(suppl 1): p. 5303-5310

32. Jia, Y., et al. (2021). Bibliometric Analysis on Global Research Trends of Airborne Microorganisms in Recent Ten Years (2011-2020). Aerosol and Air Quality Research, 21(2), 200497

33. Li, D., et al. (2020). Characterizing Hotspots and Frontier Landscapes of Diabetes-Specific Distress from 2000 to 2018: A Bibliometric Study. BioMed Research International, 2020, 8691451

34. van Eck, N. J., et al. (2010). A comparison of two techniques for bibliometric mapping: Multidimensional scaling and VOS. Journal of the American Society for Information Science and Technology, 61(12), 2405-2416

35. Waltman, L., Van Eck, N. J., \& Noyons, E. C. M. (2010). A unified approach to mapping and clustering of bibliometric networks. Journal of Informetrics, 4 
36. Rizzi, F., van Eck, N. J., \& Frey, M. (2014). The production of scientific knowledge on renewable energies: Worldwide trends, dynamics and challenges and implications for management. Renewable Energy, 62, 657-671

37. Khalil, G. M., \& Gotway Crawford, C. A. (2015). A Bibliometric Analysis of U.S.-Based Research on the Behavioral Risk Factor Surveillance System. American Journal of Preventive Medicine, 48(1), 50-57

38. Chen, C., Ibekwe-Sanjuan, F., \& Hou, J. (2010). The Structure and Dynamics of Co-Citation Clusters: A Multiple-Perspective Co-Citation Analysis.

39. Martiny, J. B. H., et al. (2006). Microbial biogeography: putting microorganisms on the map. Nature Reviews Microbiology, 4(2), 102-112

40. Caporaso, J. G., et al. (2010). QIIME allows analysis of high-throughput community sequencing data. Nature Methods, 7(5), 335-336

41. Chen, C. (2006). CiteSpace II: Detecting and Visualizing Emerging Trends. Journal of the American Society for Information Science \& Technology, 57(3), 359-377

42. Guozhong, D., et al. (2014). Microblog Burst Keywords Detection Based on Social Trust and Dynamics Model. Chinese Journal of Electronics, 23(04), 695-700

43. Ladau, J., \& Eloe-Fadrosh, E. A. (2019). Spatial, Temporal, and Phylogenetic Scales of Microbial Ecology. Trends in Microbiology

44. Florido-Benítez, L. (2021). The effects of COVID-19 on Andalusian tourism and aviation sector. Tourism Review, ahead-of-print(ahead-of-print)

45. Gregory, C. J., et al. (2012). Ultra-high-throughput microbial community analysis on the IIlumina HiSeq and MiSeq platforms. The ISME journal, 6(8)

46. Giatsis, C., et al. (2016). Probiotic legacy effects on gut microbial assembly in tilapia larvae.Scientific Reports, 6(1)

47. McMurdie, P. J., \& Holmes, S. (2013). phyloseq: An R Package for Reproducible Interactive Analysis and Graphics of Microbiome Census Data.PLOS ONE, 8(4)

48. Jia, Z., \& Conrad, R. (2009). Bacteria rather than Archaea dominate microbial ammonia oxidation in an agricultural soil. Environmental Microbiology, 11(7), 1658-1671

49. Gregory, C. J., et al. (2010). QIIME allows analysis of high-throughput community sequencing data.Nature methods, 7(5)

50. Nguyen, N. H., et al. (2016). FUNGuild: An open annotation tool for parsing fungal community datasets by ecological guild.Fungal Ecology, 20

51. Tydecks, L., et al. (2018). Spatial and topical imbalances in biodiversity research. PLOS ONE, 13(7), e0199327

52. Zhu, X., et al. (2021). Bibliometric and Visual Analysis of Research on the Links Between the Gut Microbiota and Depression From 1999 to 2019.Frontiers in Psychiatry, 11(1532)

53. Wang, S. Q., et al. (2020). A Bibliometric Analysis Using CiteSpace of Publications from 1999 to 2018 on Patient Rehabilitation After Total Knee Arthroplasty. Medical science monitor: international 
medical journal of experimental and clinical research, 26: p. e920795-1

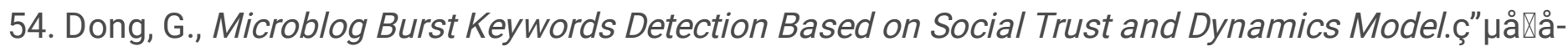
|æŠ¥:èı $\pm æ-\neq c ̧ \%{ }^{\wedge}, 2014(23):$ p.700

55. Fierer, N., \& Jackson, R. B. (2006). The diversity and biogeography of soil bacterial communities. Proceedings of the National Academy of Sciences, 103(3)

56. Martiny, J. B. H., et al. (2006). Microbial biogeography: putting microorganisms on the map.Nature Reviews Microbiology, 4(2)

57. L, L. C., et al. (2009). Pyrosequencing-based assessment of soil pH as a predictor of soil bacterial community structure at the continental scale.Applied and environmental microbiology, 75(15)

58. Vieublé-Gonod, L., et al. (2009). Spatial and temporal heterogeneity of soil microorganisms and isoproturon degrading activity in a tilled soil amended with urban waste composts.Soil Biology and Biochemistry, 41(12)

59. Sascha, K., Claudia, L., \& Peter, F. (2009). Spatial heterogeneity of methanotrophs: a geostatistical analysis of pmoA-based T-RFLP patterns in a paddy soil.Environmental microbiology reports, 1(5)

60. B, W. B. (2014). and J.M. M, The microbial nitrogen cycle.Frontiers in microbiology, 5

61. Choudhury, E., \& Kennedy, I. R. (2004). Prospects and potentials for systems of biological nitrogen fixation in sustainable rice production. Biology and Fertility of Soils, 39(4), 219-227

62. Lipponen, M., Suutari, M. H., \& Martikainen, P. J. (2002). Occurrence of nitrifying bacteria and nitrification in Finnish drinking water distribution systems. Water Research, 36(17), 4319-4329

63. Nakata, K., \& Doi, T. (2006). Estimation of primary production in the ocean using a physicalbiological coupled ocean carbon cycle model (21 vol., pp. 204-228). Environmental Modelling \& Software. 2

64. Santos, E. C., et al. (2014). Artificial neural network modeling of microbial community structures in the Atlantic Forest of Brazil. Soil Biology \& Biochemistry, 69(1), 101-109

65. Sadeghi, J., et al. (2021). Microbial community and abiotic effects on aquatic bacterial communities in north temperate lakes. Science of The Total Environment, 781(1), 146771

66. Zhao, S., et al. (2019).Biogeographical distribution of bacterial communities in saline agricultural soil. Geoderma, 361

67. Cui, H., et al. (2013). Spatial and temporal variation in enterococcal abundance and its relationship to the microbial community in Hawaii beach sand and water. Appl Environ Microbiol, 79(12), 36013609

68. Song, Y., et al. (2019). Biochar decreases soil N 20 emissions in Moso bamboo plantations through decreasing labile $\mathrm{N}$ concentrations, $\mathrm{N}$-cycling enzyme activities and nitrification/denitrification rates. Geoderma, 348, 135-145

69. J, S. C., \& Mark, O. A. (2009). Advantages and limitations of quantitative PCR (Q-PCR)-based approaches in microbial ecology.FEMS microbiology ecology, 67(1) 
70. Peter, M. W., \& R, G. M. (2015). Quantifying the relative roles of selective and neutral processes in defining eukaryotic microbial communities. The ISME journal, 9(9)

\section{Tables}

Table 1. Top 10 productive Journals in term of citations

\begin{tabular}{ccccc}
\hline Rank & Journal Titles & Citation & Documents & $\begin{array}{c}\text { Year 2020 Impact } \\
\text { Factor }\end{array}$ \\
\hline 1 & Applied and Environmental & 14861 & 184 & 4.016 \\
2 & Microbiology & 11133 & 88 & 9.412 \\
3 & PNAS $^{*}$ & 9764 & 168 & 9.180 \\
4 & ISME $^{*}$ journal & 9193 & 24 & 42.778 \\
5 & Nature $_{6}$ & 8816 & 158 & 4.933 \\
7 & Fnvironmental Microbiology $^{*}$ Microbiology Ecology & 8642 & 216 & 3.675 \\
8 & Soil Biology \& Biochemistry & 6631 & 159 & 5.795 \\
9 & New Phytologist & 6107 & 68 & 8.512 \\
10 & Frontiers in Microbiology & 5689 & 290 & 4.235 \\
\hline
\end{tabular}

Note: PNAS*; Proceedings of the national academy of sciences, ISME*; International society for microbial ecology, FEMS*; Federation of European Microbiological Societies.

Table 2. Top 6 co-citation clusters based on frequency

\begin{tabular}{ccccc}
\hline Cluster ID & Size & Silhouette & Label (LLR) & Mean Cited Year \\
\hline 0 & 227 & 0.876 & biogeographic pattern & 2015 \\
1 & 201 & 0.869 & fungal communities & 2012 \\
2 & 194 & 0.817 & ectomycorrhizal fungi & 2010 \\
3 & 154 & 0.931 & bacterial 16 & 1998 \\
4 & 147 & 0.928 & chesapeake bay & 2002 \\
5 & 144 & 0.869 & spatial scaling & 2005 \\
\hline
\end{tabular}

\section{Figures}




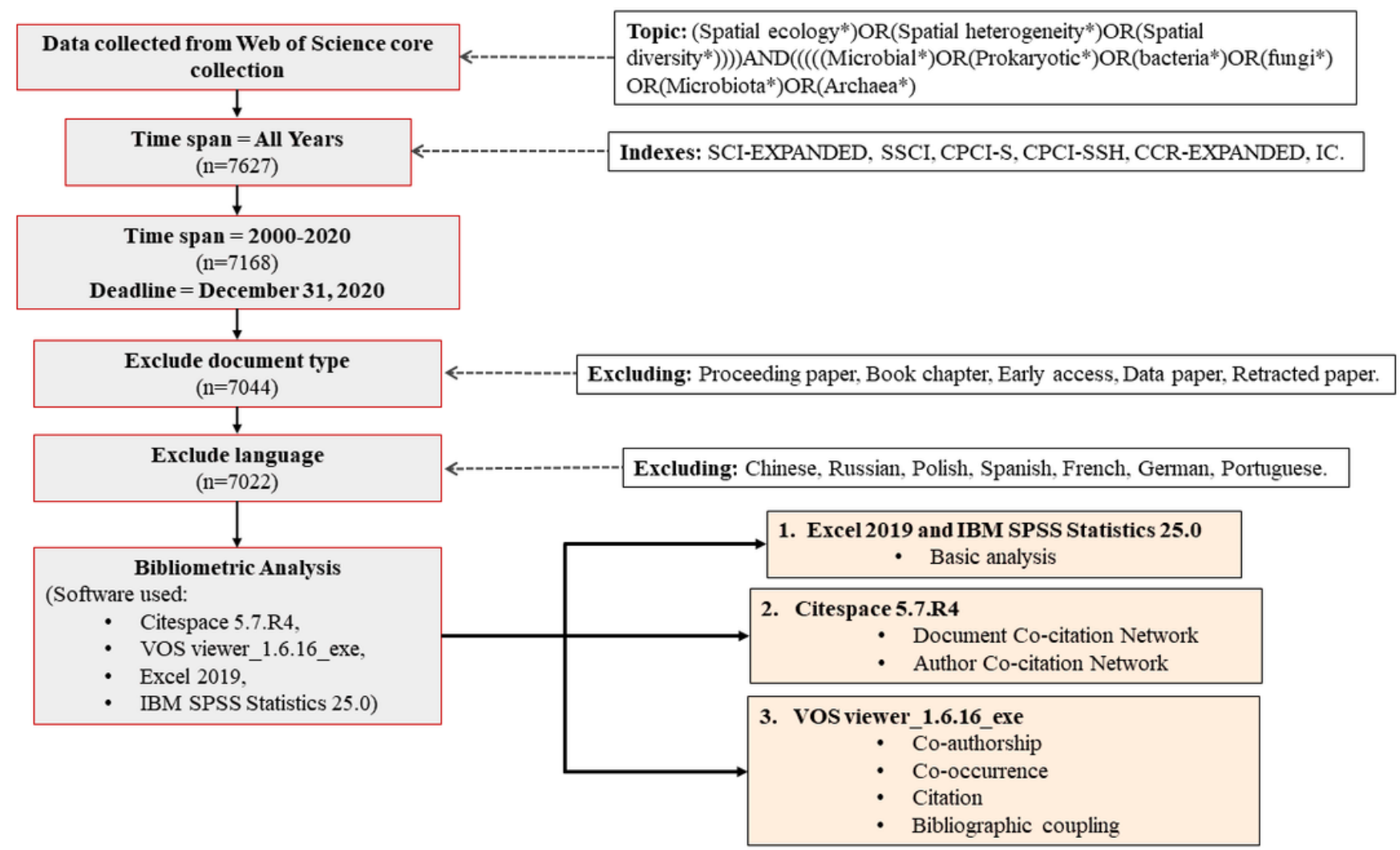

\section{Figure 1}

Flow chart for refining and retrieving literature data from web of science core collection.

$\mathbf{A}$

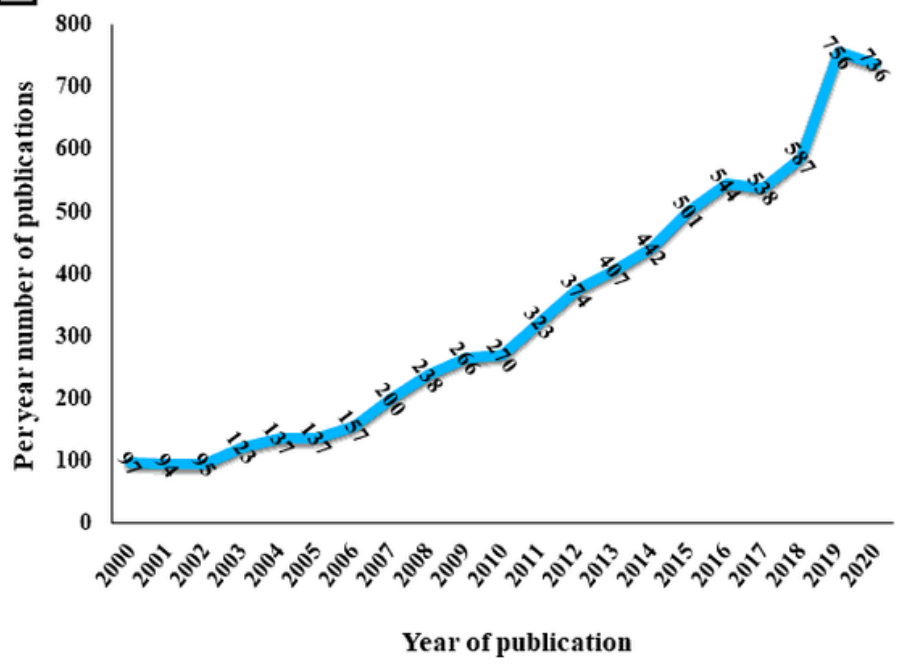

B

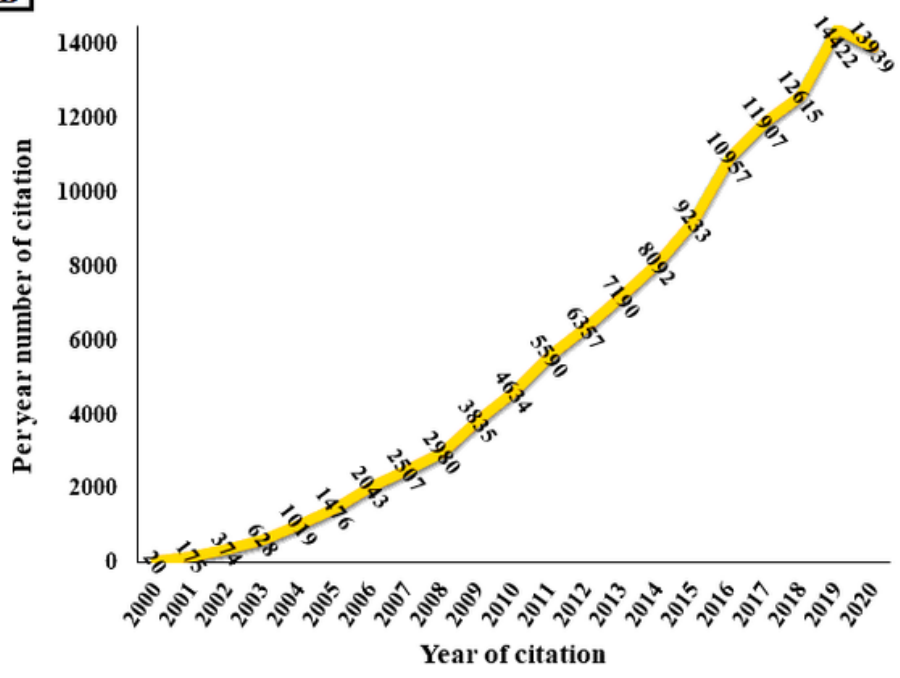

Figure 2

Temporal trends of spatial microbial ecology publication and citation per year from 2000 to 2021 in Web of Science database. 


\section{Figure 3}

Co-authorship network of top 100 authors (A) Documents published (B) Citation analysis.

\section{Figure 4}

(A) Co-citations network of cited authors. Note: The network was created using VoSviewer based on samples ( $\mathrm{N}=7022)$. (B) Authors with the strongest citation bursts in publications on spatial microbial ecology from 2000 to 2020 .

\section{Figure 5}

Co-citation network analysis of reference literature on Spatial Microbial Ecology from 2000-2020.

\section{Figure 6}

(A) Keywords co-occurrence network clustering. (B) Top 50 Keywords with strongest citation bursts in publications from 2000-2020.

\section{Supplementary Files}

This is a list of supplementary files associated with this preprint. Click to download.

- Supplementryinformation100122.docx 